\title{
Phylogenetic relationships among the Anopheles hyrcanus species group based on the degree of hybrid development and comparison with phylogenies by other methods
}

\author{
BY Kenji TAKaI and Tozo Kanda \\ Department of Medical Zoology, St. Marianna University School of Medicine, \\ 2095 Sugao, Miyamae-ku, Kawasaki 213
}

(Received July 10, 1986)

\begin{abstract}
Phylogenetic relationships among 7 members of the Anopheles hyrcanus species group of East Asia which contains vectors of human parasitic diseases were studied based on the development of interspecific hybrids; they were compared with phylogenetic relationships obtained from morphological and biochemical works. A study of Takai et al. (1984) on postmating reproductive isolation between 7 members of the An. hyrcanus species group demonstrated various degrees of development among hybrids. It is proposed in this study that hybrid development is correlated with phylogenetic relationship. An index of hybrid development was introduced and was applied to classification of the parental members. It was compared with a classification based on the morphological data of Harrison (1972) which were reanalyzed in this study. The data for polypeptide differences disclosed by two-dimensional gel electrophoresis conducted by Imajoh (1981) were reanalyzed to construct a classification, which was compared with the present results. A common clustering among the three classifications was a grouping of $A n$. argyropus, $A n$. nigerrimus, and An. nitidus. Laboratory colonies of this subgroup were difficult in free-mating in cages and preferred to feed on man, whereas colonies of the other species freely mated in cages and preferred to blood-feed on mice, except that $A n$. crawfordi did not freely mate in cages. Relation of the vector members to the classification is discussed. A revision in the UPGMA method is also presented.
\end{abstract}

\section{INTRODUCTION}

The Anopheles hyrcanus species group contains malaria and filariasis vectors and is distributed in East Asia. Its taxonomy was extensively studied by Reid $(1953,1963,1968)$. Contributions of Harrison $(1972,1973)$ and Harrison et al. (1973) were of importance. Phylogenetic relations constitute the backbone of taxonomy and supply evolutionary views on the organisms; these authors also proposed classifications of the An. hyrcanus species group based on morphological similarity.

On the other hand, Kanda and Oguma made genetic work on this group (Kanda and Oguma 1977a, 1977b; Oguma 1976, 1978). It was impressive that they could find a new species, An. engarensis, which is reproductively iso- 
lated from An. sinensis (Kanda and Oguma 1978). Among pairs of hybridization between members of the An.hyrcanus group, there were differences in the degree of postmating reproductive isolation, for instance, $A n$. engarensis produced $\mathrm{F}_{1}$ hybrids with $A n$. sinensis with normal hatchability (Oguma 1978) but An. sineroides yielded hybrids with An. sinensis mostly dying at the larval stage (Kanda and Oguma 1977a). Kanda et al. (1981b) made hybridization experiments with other members. These studies were extended by Takai et al. (1984), which showed that there was marked variation in the degree of postmating reproductive isolation among different hybridization pairs.

The purpose of the present study is to obtain phylogenetic relationships among 7 members of the An. hyrcanus species group based on the degree of reproductive isolation, hybrid development studied by Takai et al. (1984). Hybridization studies have been accumulated in mosquitoes (Kitzmiller et al. 1967; Davidson et al. 1967; Kanda et al. 1981a), but phylogenetic relationships have not yet been dealt with quantitatively. In order to evaluate this phylogeny, Harrison's (1972) morphological data were reanalyzed from the standpoint of numerical taxonomy and a phylogeny was constructed. Furthermore, Imajoh's (1981) biochemical data were reanalyzed to obtain a phylogeny. The latter two phylogenetic relationships were compared with the results from the hybrid development. The relation of the three vector species $A n$. nigerrimus, $A n$. sinensis, and An. lesteri to location in these three classifications was discussed.

\section{MATERIALS AND METHODS}

An index of hybrid development, the similarity index, and classification

Table 2 of Takai et al. (1984) was the source of data. Hybridization was made between 7 members of the An. hyrcanus species group, An. argyropus (A), An. nigerrimus (N), An. nitidus (I), An. sinensis (S), An. lesteri (L), $A n$. peditaeniatus (P), and $A n$. crawfordi (C) (letters in parentheses denote abbreviated names). It was shown that there was marked variation in the degree of hybrid development among different hybridization pairs. In the present analysis, 7 metamorphic stages, egg, developed embryo, 1st, 2nd, and 4th larval instars, pupa, and adult are assigned the values $0,0.5,1,2.5,4,5$, and 6, respectively, denoted by $k_{i}(i=0,1, \cdots, 6)$. Let $n_{k_{i}}$ be the number of progeny at the stage of the value $k_{i}$. Then an index of development, the average attained stage, $a$ among the progeny is defined as:

which reduces to

$$
a=\left[\sum_{i=0}^{5} k_{i}\left(n_{k_{i}}-n_{k_{i+1}}\right) / n_{0}+k_{6} n_{6} / n_{0}\right] / 6,
$$




$$
a=\left[\sum_{i=1}^{5} k_{i}\left(v_{k_{i}}-v_{k_{i+1}}\right)+k_{6} v_{6}\right] / 6
$$

where $v_{k_{i}}$ designates the survival rate from egg to the stage $k_{i}$. The value of $a$ ranges between 0 and 1; death of all the progeny at egg means $a=0$, and when all the progeny reach the adult stage, it becomes that $a=1$. For a cross where no progeny were counted at some stages, for example, of the values $k_{j+1}$ and $k_{j+2}$, the average $\sum_{l=j}^{j+2} k_{l} / 3$ was used in place of $k_{j}$, omitting the summation for $i=j+1$ and $j+2$ in $a$. Since the number of 3rd instar larvae was not counted, the average $(2+3) / 2=2.5$ was used as $k_{3}$. For a cross which lacked in counting the numbers of the 2 nd and 4 th instars, $(1+2+3+4) / 4=$ 2.5 was substituted for $k_{2}$, neglecting $k_{3}$.

Since most of the reciprocal crosses showed asymmetry in the value of $a$, their harmonic mean was used to represent the pair of $a$ 's. When the degree of discrepancy between the two values, $x_{1}$ and $x_{2}\left(x_{1} \geqq x_{2}\right)$, is measured by $d=$ $\left(x_{1}-x_{2}\right) /\left(x_{1}+x_{2}\right)$ as in the Charles-Stalker isolation index (Stalker 1942), their harmonic mean has a factor $d$ in itself: $\ddot{x}=2 /\left(1 / x_{1}+1 / x_{2}\right)=x_{1}(1-d)$. It is then standardized by the values of the control intermember crosses: $\alpha_{i j}=2 \tilde{a}_{i j} /\left(\tilde{a}_{i i}+\right.$ $\tilde{a}_{j j}$ ) where $\tilde{a}_{i j}$ is the harmonic mean of $a$ 's of the crosses between the $i$ th and $j$ th member species. By putting $j=i$, then $\alpha_{i i}=1$. The range of $\alpha$ is $0 \leqq \alpha \leqq 1$ if there is no heterosis. Thus $\alpha$ could be used as a similarity index between parental species; a similarity matrix of order $7 \times 7$ among the 7 members of the An.hyrcanus group was obtained. One of the popular clustering methods, the UPGMA method (Sneath and Sokal 1973), was applied to depict a dendrogram.

\section{Reanalysis of the data of Harrison (1972) on morphological similarity}

Harrison (1972) presented differences in 9 characters of 10 members of the An. hyrcanus species group in his Table 1. Part of the data were reanalyzed from the standpoint of numerical taxonomy (Sneath and Sokal 1973). Characters were converted into two-state characters. Harrison's original character no. 5 was qualitative, then it was coded nonadditively. Other characters were quantitative, then they were coded additively (Sneath and Sokal 1973).

Information analysis (Williams et al. 1966; Sneath and Sokal 1973) was suited for classification of the data because it could incorporate the variation due to intraspecific polymorphism. Since any data on the actual combination of Harrison's original characters over individuals was lacking, the analysis was applied to the full combination of them. For instance, argyropus (A) was represented by a group of $3 \times 5 \times 3=45$ individuals. Let $t_{x}$ be the number of OTU's (operational taxonomic units) in taxon $x$ and $n$ be the number of characters tested. For data coded by two-state characters, the information 
of taxon $x$ can be defined as

$$
H_{x}=n t_{x} \ln t_{x}-\sum_{i=1}^{n}\left[m_{i x} \ln m_{i x}+\left(t_{x}-m_{i x}\right) \ln \left(t_{x}-m_{i x}\right)\right]
$$

where $m_{i x}$ is the number of OTU's in taxon $x$ possessing the + (present) state in character $i$ (Sneath and Sokal 1973). The procedure of the information analysis is to find the smallest value of $\Delta H=H_{x+y}-H_{x}-H_{y}$, which is the change in $H$ resulting from combining $x$ and $y$ taxa. The smaller the information of a group of taxa is, the more homogeneity does it have. Thus clustering could be made according to the degree of information of the taxa, which allowed the construction of a dendrogram.

\section{Reanalysis of the data of Imajoh (1981) on similarity of polypeptides}

Application of O'Farrell's (1975) two-dimensional gel electrophoresis (2DE) for the 7 members of the An. hyrcanus species group which were dealt with by Takai et al. (1984) was successful in finding mobility differences of polypeptides among the members (Imajoh 1981). He introduced a distance measure due to the polypeptide differences. But his Table 2 could be simply interpreted as being that the 43 variable spots were grouped into polypeptides coded by 15 gene loci. Based on this interpretation, Nei's (1972) genetic distances $(D)$ between any two members $x$ and $y$ were calculated as $D=$ $-\ln J$ where

$$
J=(1 / n) \sum_{j}^{n} \sum_{i}^{m_{j}} x_{i} y_{i} / \sqrt{(1 / n) \sum_{j}^{n} \sum_{i}^{m_{j}} x_{i}{ }^{2}(1 / n) \sum_{j}^{n} \sum_{i}^{m_{j}} y_{i}{ }^{2},}
$$

in which $x_{i}$ and $y_{i}$ are the frequencies of the $i$ th allele in $x$ and $y$, respectively, $m_{j}$ is the number of alleles at locus $j$, and $n$ is the number of loci. The distance matrix of the 7 members was used to make a dendrogram by means of the UPGMA method.

All computation was made by using a SHARP PC-1300S and a PC-1500.

\section{RESULTS AND ANALYSIS}

Index of development, the similarity index, and classification

The $a$ and $\alpha$ values were calculated from Table 2 of Takai et al. (1984) and presented in Table 1. $\alpha$ for I-N is larger than 1 owing to heterosis. Other $\alpha$ 's are all less than 1 from 0.732 for C-L to 0.065 for N-C. From considerations about $\alpha$ in the Discussion, we propose to use $\alpha$ as a phylogenetic similarity between the parental members. We obtained a similarity matrix of $\alpha$ of order $7 \times 7$ among the members of the An. hyrcanus group. Fig. 1a shows the dendrogram constructed by the UPGMA method. I and $\mathrm{N}$ were as close 


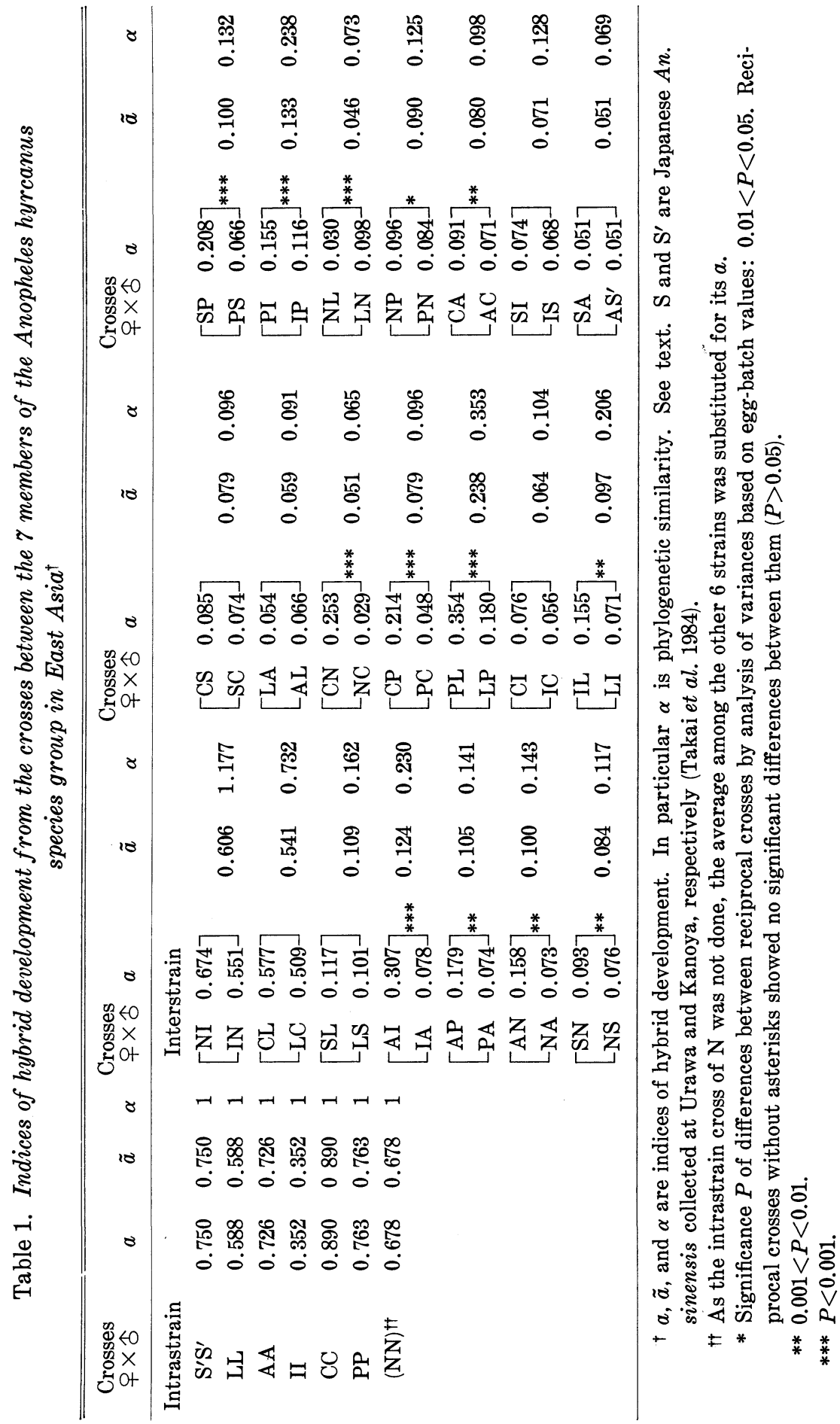




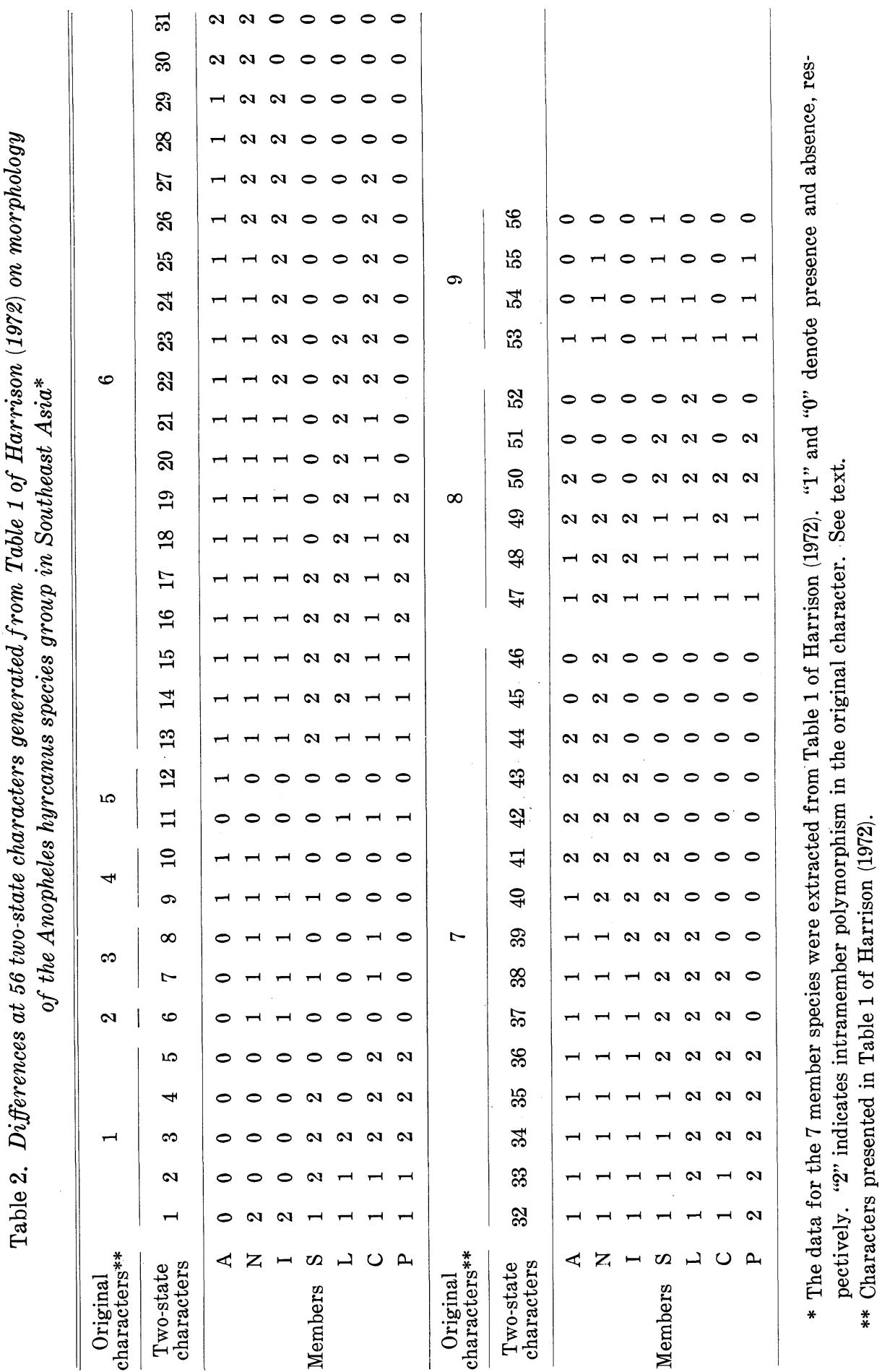



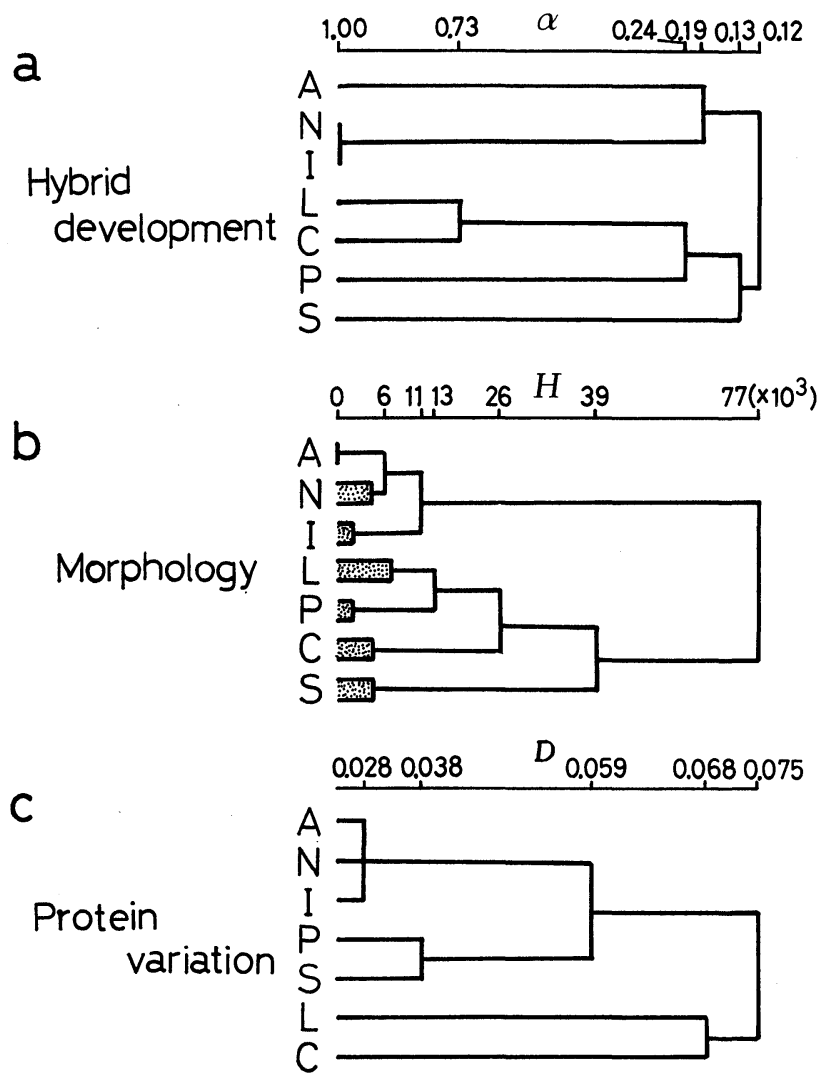

Fig. 1. Dendrograms of the 7 members of the Anopheles hyrcanus species group in East Asia.

a: From hybrid development of Table 1 by UPGMA clustering. Original data in Takai et al. (1984). The abscissa is in phylogenetic similarity $\alpha$ scale. (N, I) had $\alpha=$ 1.177, which would give negative branching for this pair, but was considered to be related at the conspecific level.

b: From morphological data of Table 2 by information analysis, the first matrix of which is shown in Table 3. Orignal data in Harrison (1972). The abscissa is in information scale $H$. Dotted columns indicate intramember polymorphism (see text).

c: From polypeptide variation detected by two-dimensional electrophoresis clustered by the UPGMA method applied to Table 7. Original data in Imajoh (1981). The abscissa is scaled in Nei's genetic distance $D$. For clustering of $(\mathrm{A}, \mathrm{N}, \mathrm{I})$ see Appendix.

as in the intramember level. The 7 members were grouped into $(\mathrm{A}, \mathrm{N}, \mathrm{I})$ and $(\mathrm{L}, \mathrm{C}, \mathrm{P}, \mathrm{S})$, and $\mathrm{S}$ was the most remote from any other. The L-C relation was less closer to each other than N-I.

Reanalysis of the data of Harrison (1972) on morphological similarity

Nine characters in Harrison's (1972) Table 1 were converted into 56 two- 
Table 3. Matrix of information $H$ (in the lower left triangle) and $\Delta H$ (in the upper right triangle), based on morphological characters in Table $2^{*}$

\begin{tabular}{rrrrrrrrr}
\hline \hline & & \multicolumn{7}{c}{$\Delta H$} \\
\cline { 3 - 9 } & & \multicolumn{1}{c}{$\mathrm{A}$} & $\mathrm{N}$ & $\mathrm{I}$ & $\mathrm{S}$ & $\mathrm{L}$ & $\mathrm{C}$ & \multicolumn{1}{c}{$\mathrm{P}$} \\
\hline & $\mathrm{A}$ & 0.22 & 1.15 & 1.22 & 3.75 & 3.57 & 2.74 & 3.91 \\
$\mathrm{H}$ & $\mathrm{N}$ & 5.76 & 4.39 & 2.75 & 14.03 & 17.63 & 10.83 & 14.69 \\
& $\mathrm{I}$ & 4.43 & 10.12 & 2.99 & 10.63 & 11.85 & 6.32 & 11.43 \\
& $\mathrm{~S}$ & 8.68 & 23.12 & 18.33 & 4.71 & 6.95 & 10.99 & 4.92 \\
& $\mathrm{~L}$ & 12.12 & 30.34 & 23.16 & 19.98 & 8.32 & 6.24 & 2.07 \\
& $\mathrm{C}$ & 7.67 & 19.92 & 14.02 & 20.40 & 19.27 & 4.71 & 6.26 \\
& $\mathrm{P}$ & 7.12 & 22.07 & 17.41 & 12.62 & 13.38 & 13.96 & 2.99 \\
\hline
\end{tabular}

* Figures should be multiplied by $10^{3}$ to give $H$ and $\Delta H$. This matrix shows the first step in the information analysis. Succeeding matrices are not presented.

state characters in Table 2. Seven out of the 10 members were analyzed. " 2 " indicates intraspecific polymorphism, that is, for example, $S$ has 4 character states in the Harrison's original character no. 1: (10000), (11000), (11100), and (11110). For classification among the members, the information analysis (see Materials and Methods) was conducted for the data of Table 2. Table 3 shows the information matrix of $H$ and $\Delta H$. Fig. $1 \mathrm{~b}$ presents the result of this procedure in the dendrogram of the 7 members. They were grouped into (A, N, I) and (S, C, L, P) clusters. Higher similarities were found in $\mathrm{A}-\mathrm{N}$ and L-P. $\mathrm{S}$ was the least closely related to any other member.

\section{Reanalysis of the data of Imajoh (1981) on similarity of polypeptides}

Table 4 is reproduced from Imajoh's (1981) Table 2. Table 5 shows the sum of polypeptides in each member of Table 4. A simple interpretation of these tables was that 15 loci were involved in this polypeptide variation and 2 spots in one of the loci of $\mathrm{C}$. An underlying hypothesis was that a polypeptide spot visualized by the $2 \mathrm{DE}$ would be a product of a locus (allelic products of a member were not separated) with an exception in $\mathrm{C}$. Then the number of a set of polypeptides would not be changed among related species. It was partly supported by the result that there were as many as 118 polypeptides which were common to all 8 members in Imajoh (1981). The following possibilities are discussed below: whether multiple spots per allele exist, polymorphism due to plural alleles is involved, or there are strain-specific duplications or deletions. Allelic variation among members were detected as follows. Allelic polypeptides were expected to locate nearer to each other on the two-dimensional gel. They might have differences due to amino acid substitutions and modifications that would result in mobility differences (Lewontin 1974). According 
Table 4. Imajoh's (1981) Table 2. Polypeptide variation by two-dimensional gel electrophoresis among 7 members of the An. hyrcanus species group*

\begin{tabular}{|c|c|c|c|c|c|c|c|c|c|c|c|c|c|c|c|}
\hline \multirow{2}{*}{$\begin{array}{l}\text { Polypeptide } \\
\text { No. }\end{array}$} & \multicolumn{7}{|c|}{ Members } & \multirow{2}{*}{$\begin{array}{c}\text { Polypeptide } \\
\text { No. }\end{array}$} & \multicolumn{7}{|c|}{ Members } \\
\hline & I & $\mathrm{N}$ & A & $\mathrm{P}$ & $\mathrm{S}$ & $\mathrm{L}$ & $\mathrm{C}$ & & I & $\mathrm{N}$ & $\mathrm{A}$ & $\mathrm{P}$ & $\mathrm{S}$ & $\mathrm{L}$ & C \\
\hline 1 & & & & & & & 0 & 23 & 0 & 0 & 0 & 0 & 0 & & 0 \\
\hline 2 & 0 & 0 & $\bigcirc$ & 0 & 0 & 0 & & 24 & & & & & & 0 & \\
\hline 3 & & & & & & 0 & 0 & 25 & 0 & 0 & 0 & & & & \\
\hline 4 & 0 & 0 & $\bigcirc$ & 0 & 0 & & & 26 & & & & 0 & 0 & 0 & 0 \\
\hline 5 & 0 & 0 & 0 & 0 & 0 & 0 & & 27 & & & 0 & & & & \\
\hline 6 & & & & & & & 0 & 28 & & & & & 0 & & \\
\hline 7 & & & & & & 0 & 0 & $29 * *$ & & & & & & & \\
\hline 8 & 0 & 0 & & & & & & 30 & & 0 & & 0 & & & 0 \\
\hline 9 & & & & 0 & 0 & & & 31 & 0 & & & & & 0 & \\
\hline 10 & & & 0 & & & & & 32 & & & & 0 & 0 & & \\
\hline 11 & & & & & 0 & & & 33 & 0 & 0 & 0 & & & 0 & 0 \\
\hline 12 & 0 & 0 & 0 & 0 & & 0 & 0 & 34 & 0 & & 0 & 0 & 0 & & \\
\hline 13 & 0 & 0 & 0 & 0 & 0 & 0 & & 35 & & 0 & & & & 0 & 0 \\
\hline 14 & & & & & & & 0 & 36 & 0 & & 0 & 0 & & & 0 \\
\hline $15^{* *}$ & & & & & & & & 37 & & 0 & & & 0 & O & \\
\hline 16 & & & & & & & 0 & 38 & & & & & & & 0 \\
\hline 17 & & & & & 0 & & 0 & 39 & & & & 0 & & & \\
\hline 18 & & & & & & 0 & & 40 & 0 & 0 & 0 & & & & \\
\hline 19 & 0 & 0 & 0 & 0 & & & & 41 & & & & & & 0 & \\
\hline 20 & 0 & 0 & & & & & 0 & $42 * *$ & & & & & & & \\
\hline 21 & & & & 0 & 0 & 0 & & 43 & & & & & 0 & & \\
\hline 22 & & & 0 & & & & & & & & & & & & \\
\hline
\end{tabular}

* An unidentified species $X$ in Imajoh's (1981) Table 2 is excluded. " $\bigcirc$ " denotes presence of the spot and the blank absence. $\mathrm{S}$ was $\mathrm{S}^{\prime}$ in Table 1.

** Present only in the unidentified species X.

Table 5. Total number of polypeptides in each member of the An. hyrcanus species group in Table 4

\begin{tabular}{cccccccc}
\hline \hline Members & $\mathrm{I}$ & $\mathrm{N}$ & $\mathrm{A}$ & $\mathrm{P}$ & $\mathrm{S}$ & $\mathrm{L}$ & $\mathrm{C}$ \\
\hline Total no. of polypeptides & 15 & 15 & 15 & 15 & 15 & 15 & 16 \\
\hline
\end{tabular}

to Steinberg et al. (1977), Comings (1979), Leigh Brown and Langley (1979), Racine and Langley (1980), and McLellan et al. (1983), differences in mobility among allelic polypeptides occurred primarily in the isoelectric focusing (IEF) direction. Under these critera, the 40 variable polypeptide spots in Imajoh's (1981) Fig. 2 could at first be divided into 24 groups as in Table 6 (3 polypeptide spots specific to unidentified species $\mathrm{X}$ were excluded). A conspicuous 
Table 6. Grouping of 40 polypeptide spots on the two-dimensional gel in Fig. 2 of Imajoh (1981)

\begin{tabular}{|c|c|c|c|}
\hline $\begin{array}{l}\text { Polypeptide } \\
\text { group no. }\end{array}$ & $\begin{array}{l}\text { Polypeptide } \\
\text { spots involved* }\end{array}$ & $\begin{array}{l}\text { No. of members } \\
\text { involved }(m)\end{array}$ & Locus no. \\
\hline 1 & 1,2 & 7 & 1 \\
\hline 2 & 3,4 & 7 & 2 \\
\hline 3 & 5,6 & 7 & 3 \\
\hline 4 & 7,8 & 4 & \multirow{2}{*}{4} \\
\hline 5 & 9,10 & 3 & \\
\hline 6 & 11,12 & 7 & 5 \\
\hline 7 & 13,14 & 7 & 6 \\
\hline 8 & $16,17,18$ & 3 & \multirow{2}{*}{7} \\
\hline 9 & 19 & 4 & \\
\hline 10 & 20,21 & 6 & \multirow{2}{*}{8} \\
\hline 11 & 22 & 1 & \\
\hline 12 & 23 & 6 & \multirow{2}{*}{9} \\
\hline 13 & 24 & 1 & \\
\hline 14 & 25 & 3 & \multirow{2}{*}{10} \\
\hline 15 & 26 & 4 & \\
\hline 16 & 27,28 & 2 & \multirow{2}{*}{11} \\
\hline 17 & 30,31 & 5 & \\
\hline 18 & 32,33 & 7 & 12 \\
\hline 19 & 34,35 & 7 & 13 \\
\hline 20 & 36,37 & 7 & 14 \\
\hline 21 & 38 & 1 & \multirow{4}{*}{15} \\
\hline 22 & 39,40 & 4 & \\
\hline 23 & 41 & 1 & \\
\hline 24 & 43 & 1 & \\
\hline
\end{tabular}

* Numbers are polypeptide nos. in Table 4.

feature in Table 6 is in the column of the number of members involved $(m)$ in polypeptide groups. One-third of the groups have 7. Since the allelic variation of polypeptides within species might be due to a relatively small number of amino acids which suffer substitution and modification, it would not involve large differences in molecular weight. However, among species, the number of amino acids might be large enough to result in changes in molecular weight. Taking this into consideration, examination of the spots in Imajoh's (1981) Fig. 2 revealed that the groups which had $m<7$ could be regrouped together to give $m=7$ as shown in the right side of the third column of Table 6. Each of the groups 4 and 5, 10 and 11, 12 and 13, and 16 and 17 had not a large amount of molecular weight differences. Groups 14 and 15, namely, spots 25 and 26 had a very large difference in the IEF direction without any molecular weight difference. And spot 26 was nearer to spot 27 
Table 7. Nei's genetic distance D among the An. hyrcanus species group based on Imajoh's (1981) data of two-dimensional electrophoretic polypeptide variation

\begin{tabular}{ccccccc}
\hline Members & $\mathrm{N}$ & $\mathrm{A}$ & $\mathrm{P}$ & $\mathrm{S}$ & $\mathrm{L}$ & $\mathrm{C}$ \\
\hline $\mathrm{I}$ & 0.023 & 0.023 & 0.046 & 0.070 & 0.070 & 0.076 \\
$\mathrm{~N}$ & & 0.038 & 0.054 & 0.070 & 0.062 & 0.068 \\
$\mathrm{~A}$ & & & 0.046 & 0.070 & 0.078 & 0.084 \\
$\mathrm{P}$ & & & & 0.038 & 0.070 & 0.076 \\
$\mathrm{~S}$ & & & & & 0.070 & 0.097 \\
$\mathrm{~L}$ & & & & & & 0.068 \\
\hline
\end{tabular}

but with differences in both isoelectric point and molecular weight. Therefore spot 26 was grouped with spot 25. Similarly, spot 19 (group 9) was grouped rather with group 8 than 10 . Groups 21 to 24 involved 5 polypeptides located nearer to each other with differences in both IEF and SDS directions. These polypeptides were not grouped otherwise to satisfy the condition that the numbers involved in the group should be 7 . The number of groups was reduced to 15 as had been expected from Table 5. As mentioned above, the total number of loci became $118+15=133$. Nei's (1972) genetic distances were calculated in Table 7 to make a dendrogram in Fig. 1c. Polypeptides 16 and 17 were tentatively treated as that each occurred in frequency 0.5 at locus 7 . All the other loci were monomorphic within strains.

\section{Comparison among the three dendrograms}

Different dendrograms would be compared in two respects: tree topology and branch lengths. Since we did not know the relation between the three indices $\alpha, H$, and $D$ used in this study, except that each was independently correlated with phylogenetic similarity, we compared them in tree topology. Using the metric $d_{R F}\left(T_{1}, T_{2}\right)$ of Robinson and Foulds (1981), a distance between any two trees $T_{1}$ and $T_{2}$, a consensus index is defined in this paper as $C I_{R F}\left(T_{1}, T_{2}\right)=1-d_{R F} / \max \left(d_{R F}\right)$ where $\max \left(d_{R F}\right)=3 n-6$ (Robinson and Foulds $1981 ; n=$ no. of OTU's $>2) . \quad C I_{R F}\left(T_{H}, T_{M}\right)=0.60, C I_{R F}\left(T_{M}, T_{P}\right)=0.53$, and $C I_{R F}\left(T_{H}, T_{P}\right)=0.60$ where $T_{H}, T_{M}$, and $T_{P}$ stand for dendrograms of hybrid development, morphology, and protein variation, respectively. Roughly speaking, the three dendrograms had $50-60 \%$ consensus between them. A common clustering among the three dendrograms was (A, N, I). Classifications which were common between at least two dendrograms were formation of 2 clusters, L-P-C closeness, $\mathrm{S}$ most remoteness to the other members between $T_{M}$ and $T_{H}$, L-C closeness between $T_{H}$ and $T_{P}$. Some conflicting points in clustering could be detected among the three dendrograms. The positions of 
$\mathrm{S}$ and $\mathrm{P}$ in $T_{P}$ were quite different from those in the others, and so were $\mathrm{L}$ and $\mathrm{C}$ in $T_{P}$. The position of $\mathrm{P}$ was different among the three.

\section{DISCUSSION}

\section{Correlation between hybrid development and phylogenetic similarity}

Hybridization experiment was employed as a useful means for evaluating the degree of phylogenetic relationship between species of echinoids (Onoda 1938), Amphibia (Kawamura 1956), Papilio (Ae 1979), Drosophila (Patterson and Stone 1952; Buzzati-Traverso and Scossiroli 1955), and Anopheles (Kitzmiller et al. 1967). In cases where the degree of development differed among hybrids, the authors related it to the degree of genetic affinities. It was typically stated in Kitzmiller et al. (1967) that "crosses which permit the most advanced development of the $F_{1}$ individuals are those indicating closest relationship." This idea will be explained at greater length as follows. Development of zygotes, after they are formed by hybridization, may be made as in the process of a test as to how much the two sets of genes from different entities have genetic harmony between them. The development will be made more smoothly in the zygote where genetic harmony is more closely attained, that is, the homology between the two sets of genes is higher. Therefore, more advanced development of the hybrids might be related with closer genetic affinity between the two parental entities.

However, we have not been able to explain why in an earlier evolutionary process two diverging entities will lose genetic harmony which is responsible for a later developmental stage. Consider sterile genes acting at various developmental stages in the hybrids. Mutation will occur in such sterile genes at random. That is, for example, a pair of entities experiencing a short period of divergence will produce hybrids sterile at the egg stage; another pair of entities diverged in a longer period will give hybrids sterile at the adult stage. Thus there seems to be a strong deviation in the phylogenetic array of entities based on hybrid development.

We might be able to retain the idea of the correlation between hybrid development and phylogenetic similarity by taking von Baer's law into account. It explains that members of the same group of organisms resemble each other more closely at an earlier stage of development, and the less they differ from each other at the adult stage, the more the developmental stage for distinction is advanced (Dodson 1960; Komai 1963). It is now generally accepted that morphological similarity is the parallel phenomenon to the genetic similarity (Mettler and Gregg 1969; Dobzhansky 1970; Lewontin 1974). That is, the degree of morphological similarity among organisms, which is utilized by taxonomists to sort them into phylogenies, also indicates a similar comparative degree of genetic similarity. (Since sexual dimorphism and mor- 
phological development generally occur in animals, comparison should be made in either sex at the same developmental stage.) Considering von Baer's law from this point, an insight may be made into the loss of genetic homology throughout the development between phylogenetically similar entities: earlier developmental stages might retain higher genetic homology and later developmental stages might at first tend to lose homology between closely related species. We may speculate on the existence of some protection mechanisms against mutation for the genes responsible for earlier developmental stages at the beginning of speciation.

The mosquito is one of the holometabolous insects, the development of which is clearly distinguished into three stages: of larva, pupa, and adult. Each of three stages is characterized by highly specialized morphological forms. To such a case, a simple application of the above discussion may not be made. Bates (1949) discussed this point in detail based on Chen (1946) and Wigglesworth (1939). He commented that von Baer's law was applicable to the mosquito if we considered the larva and adult as two different organisms. In larval development first-instar larva was, indeed, apt to show more general characters than the later stages but in some cases different species had identical adults with distinguishable larvae.

The genetic changes include changes of a vast array of characters disclosed by available technical methods. Morphology has turned out to be one of the more useful methods for detection of relative genetic differences among organisms as mentioned above. Von Baer's law is applicable to genetical morphological characters which are expressed, however, only as one aspect of gene action. Changes in the physiological characters are also important for recognition of specific differentiation. In the speciation theory incipient species have physiological differentiation to some extent, starting to establish reproductive isolation among them (Lewontin 1974). The earlier stage of speciation would or would not be accompanied with changes of morphological characters, but would inevitably be associated with changes of physiological characters. Hence we might have been able to explain how it happens that species with morphologically identical adults have distinguishable larvae; if physiological characters are also taken into account, such adults will surely have different traits. Development of organisms is not only a morphological change but is also and essentially a physiological change. Therefore, von Baer's law may be applicable to holometabolous insects including mosquitoes and correlation between the hybrid development and phylogenetic similarity discussed above will hold major significance among them.

Another point that would complicate the interpretation of developmental differences among hybrids from interspecific crosses was non-reciprocality. When cross-average values were compared between reciprocal crosses, nearly two-thirds of pairs of $a$ 's (13 out of 21) displayed significant non-recipro- 
cality at least at the $5 \%$ level in analysis of variance (see Table 1). Such nonreciprocality is common in hybridization experiments (see Bryan 1973). It might be resulted from differential combinations of the three factors: cytoplasm, sex chromosomes, and autosomes. Two interpretations were possible about its relation to the phylogenetic similarity between the parental entities of the hybrids. (1) A lower index of development in one of the reciprocals was caused by some exceptional factors and the closer affinity in the other reciprocal indicated their true relation. (2) The reciprocal cross of a lower index did indicate their relation showing a reduction of the effects of the factors responsible for their hybrid production and the other reciprocal showed only a relic of the cooperation of their genes in the hybrid zygotes. We took the way of hypothesizing a third alternative between the two.

\section{Index of phylogenetic similarity}

From hybridization experiments among Papilio, Ae (1979) proposed a differentiation index (DI) between parental entities: $\mathrm{DI}=100-(c+e+f) / 3$ where $c, e$, and $f$ denoted hatchability, categorized sex ratio, and categorized fertility in percentage. In this study even NI, LC, and CL which showed high hatchability and survival rate from egg to adult were sterile (Takai et al. 1984). Thus in 33 out of 42 crosses fertility factor was 0 and in other crosses survival rates from egg to adult were very low. It might be doubtful that most hybrids were fertile. Sex ratio distortion was common when hybrid adults were obtained, however, in only 6 crosses the factor was non-zero. Therefore, to the examination of the effect of incorporating both factors in the similarity index, the data of Takai et al. (1984) was not suited. Though the use of sex ratio distortion in a similarity index seemed to be originated in Haldane (1922), he did not mention the relation between the degree of sex ratio distortion in the hybrid and phylogenetic affinity of its parents. Further data are required to incorporate it into a similarity index.

\section{Phylogenetic relationships based on morphology}

Harrison (1972) presented a classification among 10 members of the $A n$. hyrcanus species group in Southeast Asia based on a deep consideration about morphological characters which should be picked up to represent specific differences. His interpretation of affinities among members of the An. hyrcanus group was $\mathrm{A}(\mathrm{NI}) \mathrm{S}(\mathrm{LCP})$ from qualitative analysis. That is, A was more closely related to $\mathrm{N}$ and the (NI) subgroup than the other members, and $\mathrm{S}$ was best located in an intermediate position between (NI) and (LCP). The present quantitative analysis largely agreed with it but showed more clearly the relation between members. 
Two-dimensional gel electrophoresis for phylogenetic studies

Imajoh (1981) analyzed the data on the protein variation by 2DE to deduce phylogenetic relationships among the An. hyrcanus group. Since he could not make a grouping of the variable allelic protein spots, he proposed a distance measure which varied from 0 to 43 (=no. of variable spots) and presented a graph of the relationships among the members.

In the present reanalysis of his data, the assumption of correspondence of one polypeptide spot to one locus left some alternative possibilities. Whether (1) multiple spots per allele, (2) polymorphism due to plural spots, or strainspecific (3) duplication or (4) deletion was involved. Among the 40 variable spots in Imajoh (1981), polypeptides 16 and 17 of $\mathrm{C}$ might be produced under the possibility (1), (2), or (3). These spots could be identified because multiple spots per allele did not seem to be accompanied with large differences in molecular weight (Wilson et al. 1977; Racine and Langley 1980; Garrels 1980). Since polypeptide 17 was common to S, polypeptide 16 would be the multiplied, allelic, or duplicated spot. The others among the 40 spots could not be considered to involve possibilities (1), (2), and (3). Also, possibility (4) did not occur among the 40 spots. Imajoh (1981) presented a figure of 118 spots which were common to all members. These 118 spots automatically rejected possibilities (3) and (4) and left possibilities (1) and (2). Conditions to produce multiple spots per allele were either that the samples had been kept in a freezer for more than 2 years or that the samples had been purified (O'Farrell 1975; Wilson et al. 1977; Racine and Langley 1980; Garrels 1980). The number of groups of multiplied spots was relatively very small. Imajoh (1981) avoided both procedures in sample preparation. Also, it might be improbable that all members had identical heterozygotes with certain different spots. Hence it might be highly probable that nearly all 118 spots each was a product of a locus.

Though the present reanalysis gave essentially the same result as Imajoh's, assignment to allelic variation uncovered Nei's $D$ range detected by $2 \mathrm{DE}$ and application of the UPGMA method enabled parallel comparison to be made with the above two phylogenies.

Comparison between the standard starch gel electrophoresis (SDE) and 2DE was made by Aquadro and Avise (1981) and Ohnishi et al. (1983) in application for the study of systematics. The level of protein divergence estimated by $2 \mathrm{DE}$ was consistently lower than that of divergence by SDE. The lower magnitude of divergence by $2 \mathrm{DE}$ has proved to be due to lower sensitivity of the technique to detect variations (McLellan et al. 1983). The observed low $D$ range between 0.023 and 0.075 has to be interpreted from this point. 
Phylogeny and the An. hyrcanus species group

Phylogenetic studies have become widely made among various organisms recently (see Sneath and Sokal 1973; Nei 1975; Berlocher 1984). Some authors of the studies utilized new experimental techniques such as SDE or 2DE in application for classification and evaluated the new techniques to be useful because the new classifications agreed well to other classifications based on morphology, chromosomal arrangements, and so on. However, such evaluation of the techniques seems invalid. Similarities measured by any techniques may have their own significance; agreement or disagreement of the classification based on new techniques to other classifications will in themselves be meaningful. There must be a higher probability that the common parts among classifications due to different techniques reflect true phylogenetic relationships. On the contrary, the conflicting parts deserve further consideration and investigation as to what evolutionary changes are involved along the lineages.

In this study three classifications agreed with in clustering $\mathrm{A}, \mathrm{N}$, and $\mathrm{I}$. Harrison's (1972) qualitative analysis of morphology specified singularity of $\mathrm{A}$ and S. It was partly confirmed in the classifications due to hybrid development as well as reanalysis of morphology. Other common parts located between at least two classifications may have the next higher probabilities to represent true relationships. Not a small number of conflicting points have to be interpreted carefully. According to Nei et al.'s (1983) model of evolution of reproductive isolation, fixation of such incompatibility genes as shown by Takai et al. (1984) can be made by random genetic drift in relatively small populations. If such being the case, there will be deviations in the phylogeny constructed from isolation data from the real phylogeny. Harrison (1979) pointed out that morphological similarity might reflect current ecological relationships rather than evolutionary history. There may be such an aspect in morphological similarity, giving rise to deviations from the real phylogeny in the morphological phylogeny. Variation detected by 2DE is known to be included in that detected by SDE (Leigh Brown and Langley 1979; Wanner et al. 1982; McLellan et al. 1983). Isozyme polymorphisms have been shown to be maintained by nearly neutral mutation and random drift (Mukai et al. 1980), which implies $2 \mathrm{DE}$ variants also undergo the same regime. This may contribute to the deviations of the phylogeny based on 2DE from reality.

Association of some characters with the present classifications is interesting. As noted by Takai et al. (1984), L, P, and S became adapted for free mating in laboratory cages, while the others did not. The latter species and $\mathrm{C}$ preferred to feed on man, but the former species on mice. Such associations may also support the classification of the group into $(\mathrm{A}, \mathrm{N}, \mathrm{I})$ and the others. No appropriate data are available to incriminate any laboratory colony as having 
vectorial capacity of malaria or filariasis. However, as noted in Takai et al. (1984), we would just like to mention that N, L, and S are vector members of the diseases. Thus vectorial traits do not seem to be associated with the classifications. This may suggest that vectorial traits are not such strictly determined genetical characters as developed by irreversable evolution but are largely dependent on environmental, ecological conditions.

Thanks are due to Dr.Y. N. Tobari and Dr. M. Matsuda, Tokyo Metropolitan University, for reading of an earlier draft of the manuscript and precious criticism and advice. We would like to thank Dr. H. Yoshimaru, Kyorin University, for his careful reading of the manuscript and useful comment. We thank The National Foreign Language Institute of Science and Technology of Japan for improving the English text. This work was supported in part by the Malaria Applied Field Research Component of the UNDP/World Bank/WHO Special Programme for Research and Training in Tropical Diseases; by the SEAMEO/TROPMED Project and JICA, both receiving funds from the Japanese Ministry of Foreign Affairs; by the Grant-in-Aid for Special Research Promotion No. 57123117 from the Japanese Ministry of Education, Science and Culture; and by a Nissan Science Foundation grant, all to T. Kanda.

\section{REFERENCES}

AE, S. A. (1979) The phylogeny of some Papilio species based on interspecific hybridization data. Syst. Entomol. 4, 1-16.

AqADRO, C. F. and Avise, J. C. (1981) Genetic divergence between rodent species assessed by using two-dimensional electrophoresis. Proc. Natl. Acad. Sci. USA 78, 3784-3788.

Bates, M. (1949) The Natural History of Mosquitoes. Macmillan, New York.

Berlocher, S. H. (1984) Insect molecular systematics. Annu. Rev. Entomol. 29, 403-433.

Bryan, J. H. (1973) Studies on the Anopheles punctulatus complex. II. Hybridization of the member species. Trans. R. Soc. Trop. Med. Hyg. 67, 70-84.

Buzzati-Traverso, A. A. and Scossiroli, R. E. (1955) The "obscura group" of the genus Drosophila. In Advances in Genetics Vol. 7 (ed. M. Demerec), pp. 47-92. Academic Press, New York.

Chen, S. H. (1946) Evolution of the insect larva. Trans. R. Entomol. Soc. London 97, 381-404.

Comings, D. E. (1979) Pe 1 Duarte, a common polymorphism of a human brain protein, and its relationship to depressive disease and multiple sclerosis. Nature 277, 28-32.

Davidson, G., Paterson, H. E., Coluzzi, M., Mason, G. F. and Micks, D. W. (1967) The Anopheles gambiae complex. In Genetics of Insect Vectors of Disease (ed. J. W. Wright and R. Pal), pp. 211-250. Elsevier, Amsterdam.

Dobzhansky, Тн. (1970) Genetics of the Evolutionary Process. Columbia University Press, New York.

Dodson, E. O. (1960) Evolution: Process and Product. Reinhold Publishing Cooperation, New York.

GARRELS, J. I. (1980) Computer-analysed two-dimensional gel electrophoresis of proteins. Trends Biochem. Sci. 5, 281-283.

Haldane, J. B. S. (1922) Sex-ratio and unisexual sterility in hybrid animals. J. Genet. 12, 101109.

HaRRison, B. A. (1972) A new interpretation of affinities within Anopheles hyrcanus complex of Southeast Asia. Mosq. Syst. 4, 73-83.

Harrison, B. A. (1973) A lectotype designation and description for Anopheles (An.) sinensis Wiedemann 1828, with a discussion of the classification and vector status of this and some other oriental Anopheles. Mosq. Syst. 5, 1-13.

Harrison, B. A., Scanlon, J. E. and Reid, J. A. (1973) A new synonym and new species name in the Southeast Asian Anopheles hyrcanus complex. Mosq. Syst. 5, 263-268. 
HARRISON, R. G. (1979) Speciation in North American field crickets: evidence from electrophoretic comparisons. Evolution 33, 1009-1023.

IмAJOH, S. (1981) Application of two-dimensional electrophoresis to the analysis of speciation in Anopheles hyrcanus complex. Seikagaku (Biochemistry) 53, 159-164 (in Japanese).

Kanda, T. and Oguma, Y. (1977a) Hybridization between Anopheles sinensis and Anopheles sineroides. Mosq. News 37, 115-117.

Kanda, T. and OGuma, Y. (1977b) Hybridization between Anopheles sinensis and Anopheles lesteri. Mosq. News 37, 118-123.

Kanda, T. and OGuma, Y. (1978) Anopheles engarensis, a new species related to sinensis from Hokkaido Island, Japan. Mosq. Syst. 10, 45-52.

Kanda, T., Takai, K., Chiang, G. L., Cheong, W. H. and Sucharit, S. (1981a) Hybridization and some biological facts of seven strains of the Anopheles leucosphyrus group (Reid, 1968). Jpn. J. Sanit. Zool. 32, 321-329.

Kanda, T., Takai, K., Oguma, Y., Chiang, G. L., Cheong, W. H., Sucharit, S., Joesoef, A. M. and Imajo, S. (1981b) Evolutionary genetics of the Anopheles hyrcanus group, the leucosphyrus group and the Pyretophorus group in East Asia and the Pacific area. In Cytogenetics and Genetics of Vectors (ed. R. Pal, J. B. Kitzmiller and T. Kanda), pp. 31-60. Kodansha, Tokyo/Elsevier, Amsterdam.

Kawamura, T. (1956) Isolation mechanisms in Amphibia. In Population Genetics (ed. T. Komai and K. Sakai), pp. 143-162. Baifukan, Tokyo (in Japanese).

Kitzmiller, J. B., Frizzi, G. and BAKeR, R. H. (1967) Evolution and speciation within the maculipennis complex of the genus Anopheles. In Genetics of Insect Vectors of Disease (ed. J. W. Wright and R. Pal), pp. 151-210. Elsevier, Amsterdam.

Komai, T. (1963) Biological Evolution Based on Genetics. Baifukan, Tokyo (in Japanese).

LakovaAra, S., Saura, A. and Falk, C. (1972) Genetic distance and evolutionary relationships in the Drosophila obscura group. Evolution 26, 177-184.

Leigh Brown, A. J. and LANGLEY, C. H. (1979) Reevaluation of level of genic heterozygosity in natural population of Drosophila melanogaster by two-dimensional electrophoresis. Proc. Natl. Acad. Sci. USA 76, 2381-2384.

Lewontin, R. C. (1974) The Genetic Basis of Evolutionary Change. Columbia University Press, New York.

McLellan, T., Ames, G. F.-L. and Nikaido, K. (1983) Genetic variation in proteins: comparison of one-dimensional and two-dimensional gel electrophoresis. Genetics 104, 381-390.

Mettler, L. E. and GregG, T. G. (1969) Population Genetics and Evolution. Prentice-Hall, Englewood Gliffs.

MukaI, T., TAChida, H. and Ichinose, M. (1980) Selection for viability at loci controlling protein polymorphisms in Drosophila melanogaster is very weak at most. Proc. Natl. Acad. Sci. USA 77, 4857-4860.

NEI, M. (1972) Genetic distances between populations. Am. Nat. 106, 283-292.

NeI, M. (1975) Molecular Population Genetics and Evolution. American Elsevier, New York.

NeI, M., Maruyama, T. and WU, C.-I. (1983) Models of evolution of reproductive isolation. Genetics 103, 557-579.

OGUMA, Y. (1976) Chromosomal polymorphism and salivary gland chromosomes of hybrids between strains of Anopheles sinensis (Diptera: Culicidae). Jpn. J. Genet. 51, 229-236.

OGuma, Y. (1978) Crossing studies among six strains of Anopheles sinensis. Mosq. News 38, 357-366.

O'FARRELL, P. H. (1975) High resolution two-dimensional electrophoresis of proteins. J. Biol. Chem. 250, 4007-4021.

Ohnishi, S., KIM, K.-W. and Watanabe, T. K. (1983) Biochemical phylogeny of the Drosophila montium species subgroup. Jpn. J. Genet. 58, 141-151.

ONODA, K. (1938) Hybridization experiments in some echinoids. Jpn. J. Genet. 13, 306-320 (in Japanese).

Patterson, J. T. and Stone, W. S. (1952) Evolution in the Genus Drosophila. Macmillan, New York.

RACINE, R. R. and LANGLEY, C. H. (1980) Genetic heterozygosity in a natural population of $M u s$ 
musculus assessed using two-dimensional electrophoresis. Nature 283, 855-857.

ReID, J. A. (1953) The Anopheles hyrcanus group in South-East Asia (Diptera: Culicidae). Bull. Entomol. Res. 44, 5-76.

REID, J. A. (1963) Notes on Anopheline mosquitoes from Malaya, with descriptions of three new species. Ann. Trop. Med. Parasitol. 57, 97-116.

REID, J. A. (1968) Anopheles mosquitoes of Malaya and Borneo. Stud. Inst. Med. Res. Malaya 31, 1-520.

Robinson, D. F. and Foulds, L. R. (1981) Comparison of phylogenetic trees. Math. Biosci. 53, 131-147.

Sneath, P. H. A. and SoKal, R. R. (1973) Numerical Taxonomy. Freeman, San Francisco.

Stalker, H. D. (1942) Sexual isolation in the species complex Drosophila virilis. Genetics 27, 238-257.

Steinberg, R. A., O'Farrell, P. H., Friedrich, U. and Coffino, P. (1977) Mutations causing charge alterations in regulatory subunits of the cAMP-dependent protein kinase of cultured S49 lymphoma cells. Cell 10, 381-391.

Takai, K., Kanda, T., Oguma, Y., Cheong, W. H., Joesoef, A. M. and Sucharit, S. (1984) Postmating reproductive isolation between 7 members of the Anopheles hyrcanus species group in East Asia. Jpn. J. Sanit. Zool. 35, 251-259.

Wanner, L. A., NeEL, J. V. and Meisler, M. H. (1982) Separation of allelic variants by twodimensional electrophoresis. J. Biol. Chem. 254, 7951-7960.

Wigglesworth, V. B. (1939) The Principles of Insect Physiology. Mathuen, London.

Williams, W. T., LAMBeRT, J. M. and LANCE, G. N. (1966) Multivariate methods in plant ecology. V. Similarity analyses and information-analysis. J. Ecol. 54, 427-445.

Wilson, D. L., Hall, M. E., Stone, G. C. and Rubin, R. W. (1977) Some improvements in twodimensional gel electrophoresis of proteins. Anal. Biochem. 83, 33-44.

\section{Appendix. A revision in the UPGMA method to give a unique solution.}

The UPGMA algorithm was described in Sneath and Sokal (1973) in detail, however, one point was neglected which would otherwise result in different relationships among OTU's. When more than two distances were the same and the minimum involving common OTU's in the clustering process, each group could be newly formed alternatively in the next step. However, in Sneath and Sokal (1973) and other literatures (ex. Lakovaara et al. 1972), we suspected that the new group was formed in this way. Consider a set of OTU's designated by alphabets. In the alphabetically ordered array of groups, the first group was mechanically picked up to form a new cluster.

Relationships among OTU's have been always discussed after UPGMA application. However, if other relationships are equally possible, interpretation will somewhat be changed. An algorithm to overcome the above defect is presented below.

Let a set of OTU's designated by non-minus integers be $J_{0}=\left\{0,1, \cdots, j_{0}\right\}$. Consider the 0th clustering step where more than 2 distances between OTU's are the same and the minimum involving a common OTU. For example, among $J_{0}=\left\{0,1, \cdots, j_{0}\right\}$, let 2 distances $U_{0,1}$ and $U_{0,2}$ be the same and the minimum (OTU 0 is common in both distances). A new group is either $(0,1)$ or $(0,2)$. Hence another alternative way is proposed: to cluster these 3 OTU's 
together, that is, to form $(0,1,2)$ as a new group. In this case a within-group average distance should be calculated as $U_{(0,1,2)}=\left(U_{0,1}+U_{0,2}+U_{1,2}\right) / 3$. Because the UPGMA picks the distance from the smallest value in the clustering step, the distance between $(0,1,2)$ and the other cluster or OTU to be joined at a succeeding step should not be smaller than $U_{(0.1,2)}$. Thus, if

$$
U_{(0,1,2),(e, \cdots, s)} \geqq U_{(0,1,2)} \quad\left(e, \cdots, s \in\left\{3,4, \cdots, j_{0}\right\}\right)
$$

holds, the grouping is acceptable and will be more appropriate than leaving the possibility of clustering either $(0,1)$ or $(0,2)$ as a new group in the next step. In general, among $J_{i}$, when $k^{\prime}(\geqq 2)$ distances, $U_{l, m}\left(l, m \in J_{i}\right)$ are the same and the minimum $\left[U_{p, q}=U_{p, r}\right.$ or $U_{p, q}=U_{q, r}(p \leqq q \leqq r,\{p, q, r\} \leqq\{l, m\})$ over more than $2 U_{l, m}$ 's $]$ and $K \equiv\{l, m\} \subseteq J_{i}$ then $\left(a_{0}, \cdots, a_{k-1}\right)$ is formed as a new group $\left(k=|K|,\left\{a_{0}, \cdots, a_{k-1}\right\}=K\right)$. ( $k^{\prime}$ is not necessarily equal to $k$.) Let $b_{u g}$ be the $g$ th original OTU's of the group $a_{u}$, and $t_{a_{u}}$ be the number of original OTU's of $a_{u}$. Then the within-group average,

$$
\begin{aligned}
& U_{\left(a_{0}, \cdots, a_{k-1}\right)}=\left(1 / \sum_{g, h} \sum_{u<v}^{\mathrm{K}} t_{a_{u}} t_{a_{v}}\right) \sum_{g, h} \sum_{u<v}^{\mathrm{K}} U_{\left(b_{u g}, b_{v h}\right)} \\
& \quad\left[u, v \in\{0, \cdots, k-1\} ; b_{u g}, b_{v h} \in J_{0} ; \mathrm{K}=k(k-1) / 2\right]
\end{aligned}
$$

should be used as the distance among this group. If

$$
\begin{aligned}
& U_{\left(a_{0}, \cdots, a_{k-1}\right),(e, \cdots, s)} \geqq U_{\left(a_{0}, \cdots, a_{k-1}\right)} \\
& {\left[e, \cdots, s \in J_{i},\left\{a_{0}, \cdots, a_{k-1}\right\} \cap\{e, \cdots, s\}=\phi\right]}
\end{aligned}
$$

holds where $(e, \cdots, s)$ is a group of OTU's or a single OTU to be joined to $\left(a_{0}\right.$, $\left.\cdots, a_{k-1}\right)$ at the $n$th step $(n>i)$, the $\left(a_{0}, \cdots, a_{k-1}\right)$ will be adopted as a new group in $J_{n}$. Otherwise, clustering has $k^{\prime}$ alternative ways, namely, each of the $k^{\prime}$ groups $(l, m)\left(l, m \in J_{i}\right)$ should be newly formed. 